\title{
Phase Errors in Phased Arrays: Implications on Forward Gain, Pointing Offset, Calibration, and Beamforming
}

\author{
Sascha Schediwy ${ }^{1}$, Danny Price ${ }^{1}$, Fred Dulwich $^{2},{\text { Ben } \text { Mort }^{2} \text {, and Christopher Williams }}^{2}$ \\ 1 Oxford Astrophysics, The University of Oxford, Keble Rd, Oxford, United Kingdom \\ 2 Oxford e-Research Centre, The University of Oxford, Keble Rd, Oxford, United Kingdom
}

\begin{abstract}
.
In this paper we show how phase errors between different analogue signal channels of a phased array affects the quality of the output phased array beam. Specifically we look at the reduction in forward gain of the array beam and the array beam pointing offset as a function of phase errors and array size. We use a combination of simulations made using the aperture array simulator OSKAR, developed at the University of Oxford, in conjunction with measured results taken from 2-PAD; a functioning, astronomical, dual-polarisation, digital beamforming, $4 \times 4$ element, aperture array prototype (Greenwood 2007) for the Square Kilometre Array (Taylor 2007) developed by a consortium of UK universities. We show that phase errors between different signal channels is specifically an important issue for broadband arrays like 2-PAD, and comment on the relative benefit of digital beamforming versus analogue beamforming engines.
\end{abstract}

\section{Introduction}

We begin this paper by reviewing how phase error relates to time delay variations in phased arrays and show how these time delay can affects the pointing of the array beam. We then introduce the OSKAR aperture array simulator and show how it can be used to generate array beam patterns from a number of aperture arrays comprised of different number of antenna elements, each with different random time delay variation on each analogue signal chain. Finally, we comment on the performance that could practically be achieved for aperture arrays of various sizes using either analogue or digital beam forming if the array were constructed using analogue components of the same quality as those currently used in 2-PAD (Ikin et al. 2010; Price et al. 2010).

\section{Time Delay Variation and Pointing Error}

An aperture array can be thought of as a collection of pairs of antenna elements separated by a distance $d$ as shown in Figure 1. Each antenna element is connected to the beamforming engine via an analogue signal chain. If there is a difference in time delay between two analogue signal chains, it will result in a pointing offset in the beam formed between these two elements. The time delay difference $\Delta t$ is related to the phase error $\Delta \phi$ by

$$
\Delta t=\frac{1}{2 \pi} \frac{\Delta \phi}{f}
$$

where $f$ is the frequency of the signal. This time delay is analogous to adding extra length to the signal travel path, so therefore it effectively acts as an offset in the pointing angle. For a pair of isotropic antennas the pointing offset $\Delta \alpha$ is given by

$$
\Delta \alpha=\sin ^{-1}\left(\frac{\Delta t \cdot c}{d}\right)
$$

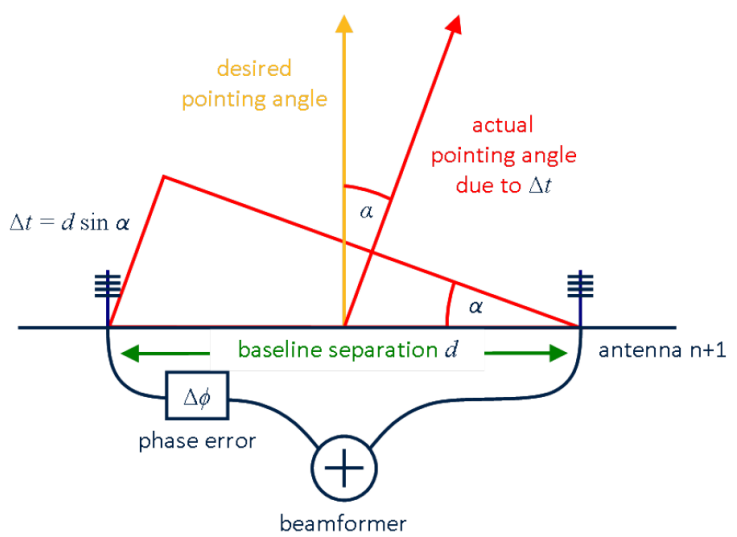

Fig. 1: Schematic representation of a pair of antenna elements in a phased array separated by a distance $d$. The actual pointing orientation is offset by an angle $\alpha$ from the desired pointing direction due to the phase difference $\Delta \phi$ between the two analogue signal chains.

where $c$ is the speed of light. However, what is of more interest is the characteristics of a beam formed from an array of a large number of antenna elements with more realistic beam profiles, For this task the OSKAR array simulator is the perfect testbed for this analysis.

\section{OSKAR Aperture Array Beam Simulations}

The OSKAR simulator is a computational package for investigating novel beamforming techniques and computational algorithms for the SKA aperture arrays (Dulwich et al. 2010). The full capabilities and functionality of OSKAR are described by Dulwich and Mort (Dulwich \& Mort 2009). OSKAR allows a user to easily create an aperture array using the graphical user interface shown in Figure 2 and Figure 3. 


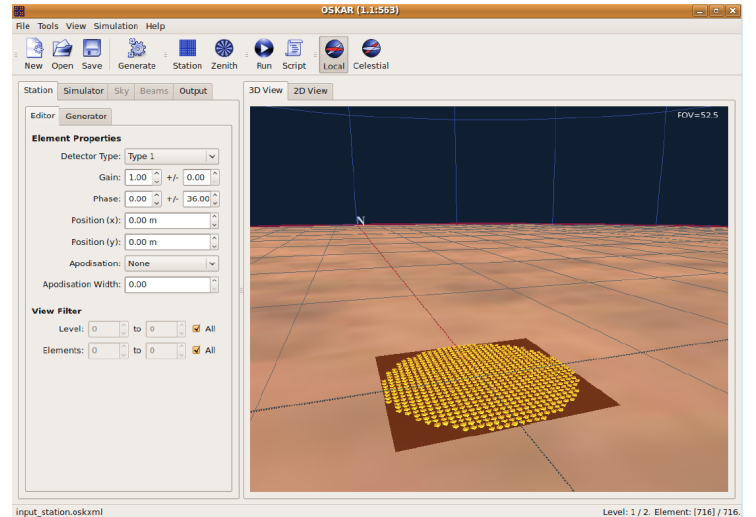

Fig. 2: A screen capture of the OSKAR graphical user interface frontend showing a model of an aperture array in the 3-D visual editor window.

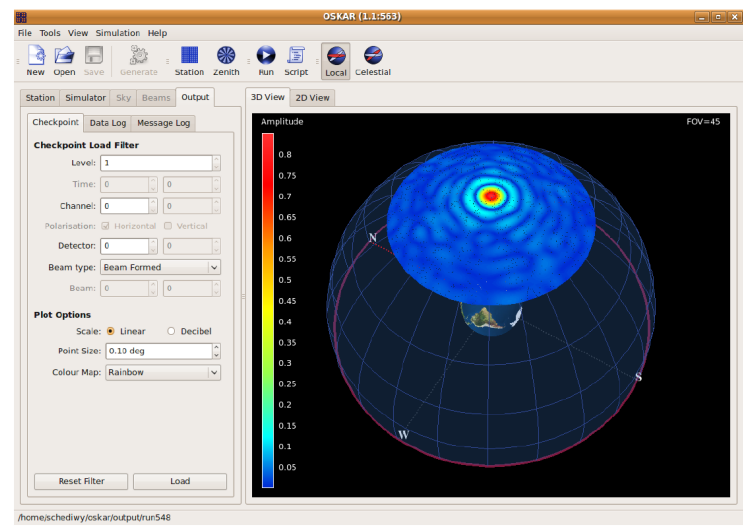

Fig. 3: A screen capture of the OSKAR graphical user interface showing a beam radiation intensity pattern of an aperture array beam formed using antenna elements with random phase errors.

A large number of parameters can be specified including; the number of antenna elements, the antenna type, the station size, the station layout, the station location on Earths surface, the apodisation function, and many others. Importantly for our purposes, it is also possible to assign an individual phase error $\Delta \phi$ to each array element. The OSKAR back-end can then compute (amongst many other things) the resultant array beam pattern.

We started our analysis by assigning a beam profile to each antenna element in the simulated aperture array. We based the beam profile on the embedded beam pattern obtained from experiments conducted with 2-PAD. We then specified a phase error value. From this value OSKAR creates a Gaussian distribution of phase errors with a standard deviation that matches this chosen value and applies these randomly to all the array elements. This reflects a real-life situation since the time delays of each analogue signal chain will be randomly distributed in Gaussian-like way across the array. We then ran the OSKAR back-end multiple times to statistically determine the typical values of forward gain and the typical range of pointing offsets associated with each configuration.

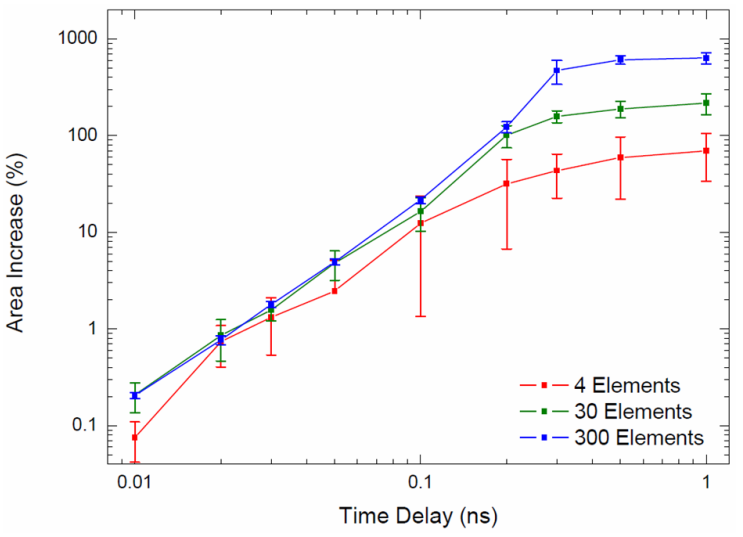

Fig. 4: Plot of the increase in aperture array effective collecting area required in order to compensate for the loss of forward gain as a result of time delay variations for three aperture array sizes; $4 \times 4$ elements, $30 \times 30$ elements, and $300 \times 300$ elements.

Perhaps a more intuitive way to express the affect of phase error on forward gain is to think about the effective colleting area of an array. Since a decrease in forward gain reduces the sensitivity of the array, this is equivalent to a decrease in collecting area. We can therefore calculate a value of effective area increase which would be required in order to counter the reduction due to the loss in forward gain. This allows us to easily compare whether it is more cost efficient to build a larger array, or to spend our time and money minimising phase errors. Figure 4 shows three plots of the required effective area increase as a function of time delay, from which we can deduce some reasonable upper limits for how precisely we need to constrain the time delays.

We can see that for small time delay variations $(0.01 \mathrm{~ns})$ the required area increase is less than $1 \%$ for all array sizes. However, as the time delay variation is increased to $0.1 \mathrm{~ns}$, an area increase of about $10 \%$ is already required. For large arrays $(300 \times 300$ elements $)$ the increase in area gets very large since the combination of partial beams from so many element-pairs drastically smears-out the forward gain of the array beam. On the other hand, for small arrays $(4 \times 4$ elements $)$ the uncertainty in the forward gain is very large. This is because only a small number of elements are phased-up to produce the array beam, so for any specific incarnation of the aperture array, the simulation can produce an array beam with drastically different properties. Therefore, if one wanted to keep the required increase in area to less than about $5 \%$ we would have to limit the standard deviation of the time delay difference in the analogue channels to less than $0.05 \mathrm{~ns}$.

As mentioned previously, another consideration is the typical range of pointing errors that might result for a given set of phase errors. Figure 5 shows the uncertainty (one standard deviation) in the range of pointing offsets as a percentage of the half-power beamwidth, as a function of time delay variation for the same three array sizes. The dotted line in Figure 5 represents a one standard deviation chance that the pointing offset of the array beam will be greater than $5 \%$ of the beamwidth. For a $4 \times 4$ element array the limit is around $0.03 \mathrm{~ns}$, for a 


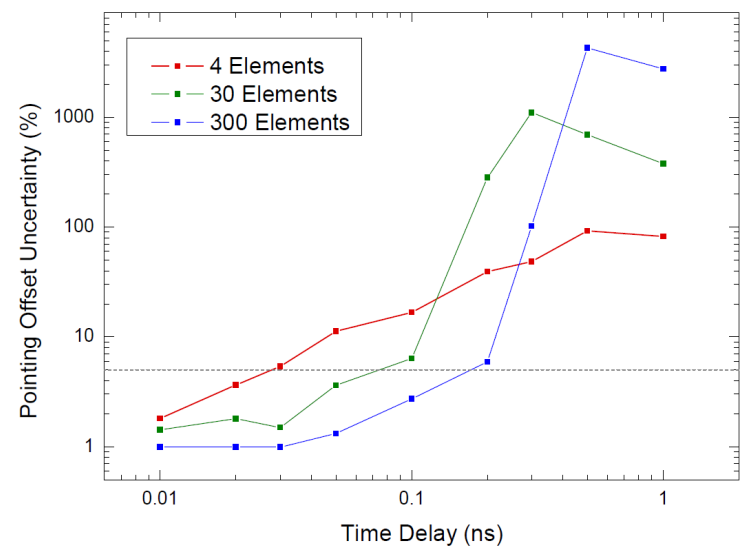

Fig. 5: Plot of the uncertainty (one standard deviation) in the range of pointing offsets as a percentage of the half-power beamwidth as a function of time delay for three array sizes; $4 \times 4$ elements, $30 \times 30$ elements, and $300 \times 300$ elements.

$30 \times 30$ array it is about $0.07 \mathrm{~ns}$, while or the $300 \times 300$ array it is about $0.2 \mathrm{~ns}$.

Therefore if one would like to construct an aperture array that requires no more than a 5\% increase in optimal collecting area, and has a pointing error of less than $5 \%$ of the beamwidth, the following limits apply: for small, (2-PAD sized) arrays, the limit of $\Delta t$ is $0.03 \mathrm{~ns}$ with the pointing being the limiting factor. For larger arrays the limiting factor becomes the loss in forward gain, thus restricting the time delay difference to $0.05 \mathrm{~ns}$.

\section{Aperture Array Calibration}

The intrinsic time delay differences between signal channels of the analogue components of 2-PAD was measured to be in the order of $1 \mathrm{~ns}$ (Price et al. 2010). As can be seen in Figure 4 and Figure 5 such differences would lead to completely ineffective array beams. In order to form close-to-optimal array beams the signals need to be calibrated.

One technique that has previously been employed is to simply alter a length of coaxial cables until the time delay difference is sufficiently. However, this is only true at one particular frequency and even if this technique could be implemented perfectly in practice there could still be residual problems.

The first issue is that this technique can be difficult and time consuming to put into practice. It would probably only be feasible to conduct this procedure once prior to the array being deployed. However, the analogue components could vary with time and temperature resulting in dynamical changes in the time delay difference. The second issue is that the time delay will not be the same across the full bandwidth of the array resulting in a residual time delay difference even if there is perfect calibration at one particular frequency. This issue becomes increasingly significant for arrays with large fractional bandwidths. For example, Figure 6 shows the ideal residual time delay difference between four analogue channels over the full RF bandwidth of 2-PAD.

A digital calibration system that channelises the frequency spectrum into many bins and performs digital corrections for

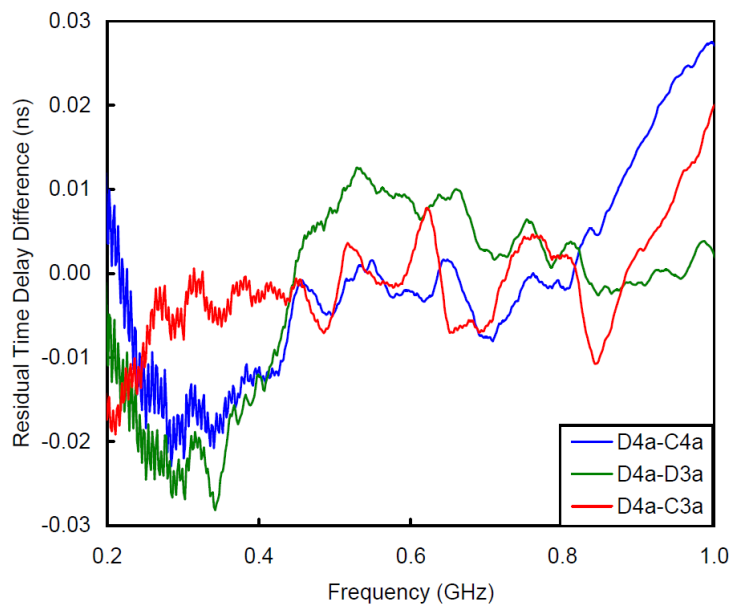

Fig. 6: Plots of the measured residual time delay differences over the entire 2-PAD RF bandwidth between four randomly chosen analogue channels.

every frequency bin can significantly reduce this residual and can be applied dynamically, thereby tackling both these issues. For example, the 2-PAD analogue to digital converter channelised the RF bandwidth into $500 \mathrm{kHz}$ wide bins, so even in areas of greatest delay gradient shown in Figure 6, the digital beamformer can reduce the residuals to less than $0.1 \mathrm{ps}$, which is only about 0.04 at $1 \mathrm{GHz}$.

\section{Conclusions}

In this paper we have shown how the time delay variation between different analogue signal channels of a phased array affects the forward gain and the uncertainty of the pointing offset of the array beam for three different sized arrays.

We found that if one would like to construct an aperture array that requires no more than a $5 \%$ increase in optimal collecting area, and has a pointing error of less than $5 \%$ of the beamwidth, the following limits apply: For small arrays the limit of $\Delta t$ is $0.03 \mathrm{~ns}$ with the uncertainty in pointing being the limiting factor. For larger arrays the limiting factor becomes the reduction in forward gain, thus restricting the time delay difference to $0.05 \mathrm{~ns}$.

We argued that a digital beamforming engine is advantageous over a more traditional analogue beamformer, due to the ability to dynamically calibrate the various analogue signal channels as a function of frequency over time. This drastically improves the residual time delay difference between channels, resulting in near optimal forward gain and pointing offset of the array beam.

Acknowledgements. 2-PAD is a joint project of The University of Oxford, The University of Manchester and The University of Cambridge. 2-PAD has been developed under the Square Kilometre Array Design Studies project, which is funded by the European Commission Sixth Framework Programme and the United Kingdom Science and Technology Facilities Council. 


\section{References}

Cordes, J.M., P. E. Dewdney, R. D. Ekers, et al., "Preliminary specifications for the Square Kilometre Array," SKA Memos, 2008.

Dewdney, P.E.F, T.J.W. Lazio, P.J. Hall, and R.T Schilizzi, "The Square Kilometer Array (SKA) radio telescope: Progress and technical directions," The Radio Science Bulletin, No. 326:419, 2008.

Dulwich, F., Mort, B., “OSKAR User Documentation,” Oxford e-Research Centre, July 2009.

Dulwich, F, B. Mort, and S. Salvini, "OSKAR: Simulating Station Beams for the SKA Aperture Array" 2010, in Proc. Wide Field Science and Technology for the SKA, Limelette, Belgium, S.A. Torchinsky et al. (eds), ASTRON, ISBN 97890-805434-5-4

Greenwood, C, "Status of pathfinder telescopes and design studies" SKA Memos, 2007.

Ikin, T.S, P.N. Wilkinson, A.J. Faulkner, et al., "Progress on Analogue Front end for 2PAD," 2010, in Proc. Wide Field Science and Technology for the SKA, Limelette, Belgium, S.A. Torchinsky et al. (eds), ASTRON, ISBN 978-90805434-5-4

Price, D., Schediwy, S., Ikin, T., Jones, M., "The 2PAD Analogue System: Characterisation, Commissioning, and Implications for Calibration and Beamforming," SKA Memo \#122, 2010, available from http://www. skatelescope.org/pages/documents_3.htm

Taylor, A.R., "The Square Kilometre Array," Proceedings IAU Symposium No. 248, 2007. 